\title{
Expression of immune checkpoint molecules in endometrial carcinoma
}

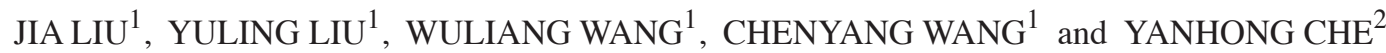 \\ ${ }^{1}$ Department of Gynecology and Obstetrics, The Second Affiliated Hospital of Zhengzhou University, Zhengzhou, \\ Henan 450014; ${ }^{2}$ Department of Gynecology and Obstetrics,Women \& Infants Hospital of Zhengzhou, \\ Zhengzhou, Henan 450000, P.R. China
}

Received September 24, 2014; Accepted August 10, 2015

DOI: $10.3892 / \mathrm{etm} .2015 .2714$

\begin{abstract}
The main obstacle in the development of an effective tumor vaccine is the inherent ability of tumors to evade immune responses. Tumors often use common immune mechanisms and regulators to evade the immune system. The present study aimed to analyze the expression levels of indoleamine 2,3-dioxygenase (IDO), programmed death-ligand (PD-L) 1, PD-L2, B7-H4, galectin-1 and galectin-3 in tissue samples from patients with endometrial carcinoma, in order to detect the immunosuppressive environment of endometrial carcinomas. The levels of IDO, PD-L1, PD-L2 and B7-H4 were analyzed by immunohistochemical methods, and the levels of galectin-1 and galectin-3 in tumor lysates were determined using ELISA. PD-L2 was expressed at low levels in the majority of tumor samples. IDO expression was detected in 38,63 and $43 \%$ of primary endometrial carcinoma, recurrent endometrial carcinoma, and metastatic endometrial carcinoma specimens, respectively. Positive expression rates for PD-L1 were $83 \%$ in primary endometrial carcinoma, $68 \%$ in recurrent endometrial carcinoma, and $100 \%$ in metastatic endometrial carcinoma, whereas B7-H4 expression was detected in $100 \%$ of both primary endometrial carcinoma and recurrent endometrial carcinoma samples, and in $96 \%$ of metastatic endometrial carcinoma specimens. The expression levels of galectin-1 and galectin-3 were not significantly different between the normal and tumor specimens. The results of the present study suggest that the interaction between PD-1/PD-L1 and B7-H4 may be a potential target for immune intervention in the treatment of endometrial carcinoma. Furthermore, the results may provide the basis for immunosuppressant therapy in the treatment of patients with uterine cancer.
\end{abstract}

Correspondence to: Dr Yuling Liu, Department of Gynecology and Obstetrics, The Second Affiliated Hospital of Zhengzhou University, 2 Jingba Road, Zhengzhou, Henan 450014, P.R. China E-mail: yulingliucn@163.com

Key words: immunosuppressant, endometrial carcinoma, immune checkpoint

\section{Introduction}

Endometrial carcinoma, which is also known as corpus carcinoma, is a common gynecological malignant tumor, with an incidence that is second only to cervical cancer(1). The five year survival rate of endometrial carcinoma is 74-91\%. Endometrial carcinoma is the fourth most common cancer in Europe and the United States, accounting for $\sim 6 \%$ of newly diagnosed tumors (2). The etiology of endometrial cancer is currently not well understood. Preliminary studies have focused on a single candidate gene or pathway, such as the phosphoinositide 3-kinase (PI3K)/AKT/mammalian target of rapamycin signaling pathway $(2,3)$; however, to uncover the full range of genes recurrently mutated in endometrial carcinoma requires whole genome sequencing of numerous patient samples. The current immunosuppressive therapy used to treat endometrial carcinoma often induces anti-tumor immune responses, including antigen-specific T cell responses (4). However, tumor evasion of the immune system poses a serious challenge to the efficacy of immunosuppressive therapies. There are numerous mechanisms by which tumors may evade the host immune system, although the mechanisms used by endometrial tumors remain elusive. A possible immune evasion mechanism includes the suppression of immune checkpoints. Immune checkpoints maintain the tolerance of the immune system to self-antigens under normal physiological conditions, and negatively regulate the immune response, in order to avoid immune injury (5). In addition, these molecules have an important role in anti-tumor immunity, of which the predominant immune checkpoints involved are cytotoxic T-lymphocyte-associated protein 4 (CTLA-4) and programmed death-ligand (PD-L) 1. At present, numerous inhibitors of these molecules have been approved for the treatment of cancer by the Food and Drug Administration, including ant-CTLA4 and anti-PD-1 (6-9).

PD-L1 and PD-L2 are members of the B7-CD28 family and ligands of programmed death receptor 1 (PD-1), which has a critical role in central $\mathrm{T}$ cell tolerance during the process of T cell development. PD-L1 is expressed in various tissues, including the placenta, cells of the heart and spleen, pancreatic island and white blood cells. Furthermore, PD-L1 has been shown to be highly expressed in tumor tissue. Conversely, the expression profile of PD-L2 is very limited; and its expression is predominantly restricted to dendritic cells (DCs) and macro- 
phages $(\mathrm{M} \Phi)$. However, the expression of these molecules in numerous immune and non-immune cells can be induced. The expression levels of PD-L2 have been shown to vary between diverse types of tumor. The interaction of PD-1 with PD-L1 has been demonstrated to occur in peripheral $\mathrm{T}$ cells in order to inhibit antigen sensitization; therefore, protecting normal tissues against immune injury. In addition, upregulation of PD-L2 has been reported to be stimulated by $\mathrm{T}$ helper cell (Th)-2 cytokines, and is itself involved in the adjustment of Th2 responses (6).

B7-H4 mRNA has previously been demonstrated to be abundant in human tissue, although its protein expression levels were relatively low (10). In addition, B7-H4 has been detected in various types of human tumor (10). The soluble B7-H4 protein can be detected in the sera of patients with tumors, and is able to inhibit the activation, proliferation, and clonal expansion of $\mathrm{CD}^{+}$and $\mathrm{CD}^{+} \mathrm{T}$ cells (11).

In the present study, the expression levels of indoleamine 2,3-dioxygenase (IDO), PD-L1, PD-L2, and B7-H4 in endometrial tumors were detected by immunohistochemical methods. IDO is a key enzyme for the regulation of adaptive immune responses; galectin-1 and -3 are involved in cancer and inflammation (12). In addition, the expression levels of galectin-1 and galectin-3 were analyzed in a uterine specimen via ELISA, in order to elucidate the immunosuppressive mechanisms of endometrial cancer, and to direct the use of immunosuppressive inhibitors for the treatment of endometrial carcinoma.

\section{Materials and methods}

Specimens. All specimens were collected in the Second Affiliated Hospital of Zhengzhou University (Zhengzhou, China). A total of 72 cases of each tumor were collected by surgical operation, from patients aged 39-74 years old, with an average age of $54.52 \pm 6.47$ years. There were 42 cases of primary endometrial carcinoma, 17 cases of recurrent endometrial carcinoma and 13 cases of metastatic endometrial carcinoma. In addition, 21 samples of normal endometrial tissue which was confirmed as benign were collected. The present study was conducted in accordance with the declaration of Helsinki, and with approval from the Ethics Committee of the Second Affiliated Hospital of Zhengzhou University. Written informed consent was obtained from all participants.

Immunohistochemical staining. For all of the specimens that required staining, the tissue was sliced to $4.0 \mu \mathrm{m}$, formalin-fixed and embedded on a glass slide. Prior to staining, the sections were dewaxed and re-hydrated using an ethanol gradient as follows: $90 \%$ ethanol, $75 \%$ ethanol, $50 \%$ ethanol and $25 \%$ ethanol, successively. The staining procedure was similar. Following heating of the sections at $42^{\circ} \mathrm{C}$ for antigen retrieval, $0.2 \mathrm{M} \mathrm{HCl}$ or $0.5 \% \mathrm{H}_{2} \mathrm{O}_{2}$ dissolved in methanol was added to the specimens to inhibit endogenous alkaline phosphatase and peroxidase activities. Subsequently, the appropriate antibodies were added. Tissue sections were visualized using an AX80 microscope (Olympus Corporation, Tokyo, Japan).

Scoring system. Scoring of each tissue section was preceded by a routine dying method, in order to authenticate the tumor tissue. The tissue sections were separated and scored based on a scoring system outlined in Ino et al (13). Initially, the tissue sections were allocated into one of three categories (1-25, 25-50 or $>50 \%$, given the scores 1,2 and 3 , respectively. These categories indicated the total number of tumor cells in the sample expressing the factor of interest. Subsequently, the tissue sections were scored according to the strength of the expression: 1 , weak; 2 , moderate; and 3, strong. The final value for each tissue section was dependent on the sum of the two scores: $0-1=0 ; 2-3=1 ; 4-5=2$ and $6=3$. Based on these scores, high and low levels of expression were distinguished. According to De Jong et al (14), the specimen with a total score of $0-1$ is considered a low score, whereas that with a total score of 2-3 is considered a high score. The score of the staining result was depended on each molecule alone.

Preparation of the lysate. Frozen tissue specimens (150-200 mg) were cut and decomposed with lysis buffer $(250 \mathrm{~mm}$ Tris- $\mathrm{HCl}$, $750 \mathrm{~mm} \mathrm{NaCl}, 0.5 \%$ SDS, $2.5 \%$ cholic acid, 5\% Igepal and $0.01 \%$ protease inhibitors; Sigma-Aldrich, St. Louis, MO, USA). Following incubation at $4^{\circ} \mathrm{C}$ for $30 \mathrm{~min}$, the lysate underwent centrifugal purification at $15,000 \mathrm{x} \mathrm{g}$ at $4^{\circ} \mathrm{C}$ for $15 \mathrm{~min}$. The concentration of protein from each specimen was determined using a Bicinchoninic Acid protein assay.

ELISA. Sheep anti-human galectin-1 [ $2 \mu \mathrm{g} / \mathrm{ml}$ in phosphate-buffered saline (PBS); AF1152; R\&D Systems, Inc., Minneapolis, MN, USA] was used. The tissue specimens were blocked with PBS solution containing $1 \%$ bovine serum albumin (Takara Biotechnology Co., Ltd., Dalian, China) at room temperature for $1 \mathrm{~h}$, after which the standard protein (recombinant galectin-1; range, 25-0.39 ng/ml; Wuhan Boster Biological Technology, Ltd., Wuhan, China) was added at room temperature for $2 \mathrm{~h}$. The concentration of lysate for analysis was $5 \mathrm{mg} / \mathrm{ml}$. Sheep anti-human immunoglobulin G (200 ng/ml; 110-AG; R\&D Systems Inc.), and the horseradish peroxidase-binding chain enzyme avidin, were used for detection. Tetramethyl benzidine substrate was added for coloration, and $2 \mathrm{M} \mathrm{H}_{2} \mathrm{SO}_{4}$ was used to terminate the reaction. The OD value was detected at $450 \mathrm{~nm}$ using an ELX80 microplate reader (BioTek Instruments, Inc., Winooski, VT, USA). Galectin-3 was detected using a commercial ELISA kit (R\&D Systems, Inc.) with only minor changes, and mouse anti-human galectin-3 (2 $\mu \mathrm{g} / \mathrm{ml}$; AF1154; R\&D Systems, Inc.) was used to detect the expression levels. The standard concentration of galectin-3 was $62.5-4,000 \mathrm{pg} / \mathrm{ml}$. The detection method was similar to that used for galectin-1.

Statistical analysis. All data were processed using GraphPad Prism 5 software (GraphPad Software, Inc., La Jolla, LA, USA). All scoring data were analyzed by the non parametric Kruskal-Wallis test, followed by Dunn's multiple comparison test. The difference was calculated by Fisher test. Multiple groups were compared with two-way analysis of variance or related sample t-test. The survival curves were compared with log-rank (Mantel-Cox) test. $\mathrm{P}<0.05$ was considered to indicate a statistically significant difference.

\section{Results}

Expression of IDO in endometrial carcinoma. Of the normal endometrial samples, $57 \%$ tested positive for the expression 
Table I. The number and positive rate of immune checkpoint molecule and indoleamine 2,3-dioxygenase (IDO) expression in various sample types.

\begin{tabular}{|c|c|c|c|c|c|c|c|c|}
\hline \multirow[b]{2}{*}{ Sample } & \multicolumn{2}{|c|}{ IDO } & \multicolumn{2}{|c|}{ PD-L1 } & \multicolumn{2}{|c|}{ PD-L2 } & \multicolumn{2}{|c|}{ B7-H4 } \\
\hline & $\mathrm{n}$ & $\%$ Positive & $\mathrm{n}$ & $\%$ Positive & $\mathrm{n}$ & $\%$ Positive & $\mathrm{n}$ & $\%$ Positive \\
\hline nlEM & 21 & 57 & 16 & 78 & 23 & 44 & 22 & 100 \\
\hline prEMCAR & 42 & 38 & 29 & 83 & 45 & 40 & 53 & 100 \\
\hline recEMCAR & 17 & 63 & 9 & 68 & 7 & 78 & 17 & 100 \\
\hline metaEMCAR & 13 & 43 & 9 & 100 & 14 & 42 & 15 & 96 \\
\hline
\end{tabular}

nIEM, normal endometrium; prEMCAR, primary endometrial carcinoma; recEMCAR, recurrent endometrial carcinoma; metaEMCAR, metastatic endometrial carcinoma; PD-L1, programmed death-ligand 1; PD-L2, programmed death-ligand 2.

of IDO (Table I and Fig. 1). In the tumor specimens, IDO was expressed in only $38 \%$ of primary endometrial carcinoma samples, $63 \%$ of recurrent endometrial carcinoma specimens, and $43 \%$ of metastatic endometrial carcinoma samples. Therefore, the percentage of tumor samples that tested positive for IDO expression was significantly lower $(\mathrm{P}<0.01)$, as compared with the normal endometrium samples. IDO was predominantly expressed in the cytoplasm, however some tumors exhibited apical expression. In addition, the infiltrating cells expressing IDO were in close proximity to the tumor vessels. IDO was highly expressed in $21 \%$ of primary endometrial cancers, indicating that blocking the expression of IDO may be a useful strategy in the treatment of primary endometrial carcinoma.

Expression of PD-L1 in endometrial carcinoma. PD-L1 was expressed in the majority of the specimens analyzed (Fig. 2), and was predominantly located in the cytoplasm. PD-L1 was expressed in $78 \%$ of normal endometrium samples, and $70-80 \%$ of tumor tissues. The analyzed specimens and their corresponding percentages are shown in Table I. As compared with the normal endometrium, the expression of PD-L1 in tumor samples was upregulated $(\mathrm{P}<0.01)$. The expression levels of PD-L1 across all samples were medium to high. Of the primary endometrial carcinomas, $72 \%$ exhibited expression of PD-L1, suggesting that intervention of the PD-1/PD-L1 axis may be a promising treatment option for patients with endometrial cancer.

Expression of PD-L2 in endometrial carcinoma. The expression profile of PD-L2 differed from that of PD-L1 (Table I). PD-L2 was expressed in $47 \%$ of normal endometrium samples. The positive percentage of tumor samples ranged from $40-78 \%$. All of the biopsy specimens were scored from low to medium, and there was no significant difference among them (Fig. 3). The present study demonstrated that PD-L2 positive cells were adjacent to tumor cells, which may indicate immune cell invasion. These immune cells were predominantly distributed in the tumor, tumor stroma and peripheral tumor. Overall, PD-L2 was highly expressed in $30 \%$ of primary endometrial carcinomas, suggesting that blocking PD-L2 may be an effective therapeutic strategy in small numbers of patients with endometrial cancer.
Table II. Expression levels of galectin-1 and galectin-3 in tumor lysates were detected by ELISA.

\begin{tabular}{lcc}
\hline Items & Galectin-1 $(\mathrm{ng} / \mathrm{ml})$ & Galectin-3 $(\mathrm{pg} / \mathrm{ml})$ \\
\hline nlEM & $8.3 \pm 1.4$ & $860 \pm 53.2$ \\
EMCAR & $7.2 \pm 1.3$ & $1780 \pm 125$ \\
nlMYOM & $12.8 \pm 2.1$ & $920 \pm 86$ \\
\hline
\end{tabular}

nlEM, normal endometrium; EMCAR, endometrial carcinoma; nlMYOM, leiomyosarcoma.

Expression of B7-H4 in endometrial carcinoma. B7-H4 was expressed in the majority of biopsy specimens (Table I). Statistical analysis demonstrated that there was no significant difference in the expression levels of B7-H4 between the normal and tumor samples (Fig. 4). In addition, B7-H4 was predominantly expressed in the cytoplasm. Identifying the infiltrating immune cells was complicated by dyeing effects. The present study demonstrated that B7-H4 was highly expressed in $90 \%$ of primary endometrial carcinomas, suggesting that B7-H4 may be an attractive candidate target for therapeutic drugs.

Detection of galectin-1 and galectin-3 levels. The expression levels of galectin-1 and galectin-3 were detected in tumor lysates from 29 endometrial cancer samples, 21 normal endometrium samples and 18 leiomyosarcoma samples via ELISA. Galectin-1 was detected in all of the samples, and there was no significant difference in the expression levels of galectin-1 or galectin-3 between all of the groups (Table II).

\section{Discussion}

In the present study, the expression levels of PD-L1, PD-L2, B7-H4, IDO, galectin-1 and galectin-3 were evaluated in endometrial carcinoma samples. Previous studies have demonstrated that the ligands of PD-1, PD-L1 and PD-L2, are expressed in numerous types of tumor, including melanoma, brain tumor, lung cancer, cancer of the urinary tract and pancreatic cancer (6-9). PD-L1 is generally expressed in $65 \%$ of soft tissue tumor cases (15). However, to the best of our current knowledge, there are few reports associating PD-L1 

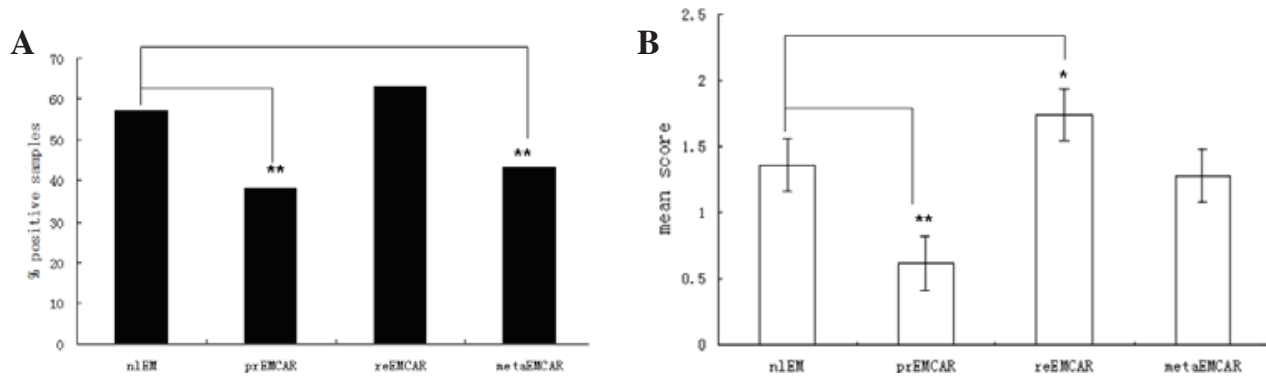

Figure 1. Expression of indoleamine 2,3-dioxygenase (IDO) in endometrial carcinoma. (A) Positive rates and; (B) relative expression levels of IDO in nlEM, normal endometrium; prEMCAR, primary endometrial carcinoma; recEMCAR, recurrent endometrial carcinoma; and metaEMCAR, metastatic endometrial carcinoma. ${ }^{*} \mathrm{P}<0.05$ and ${ }^{* *} \mathrm{P}<0.01$ vs. the control.
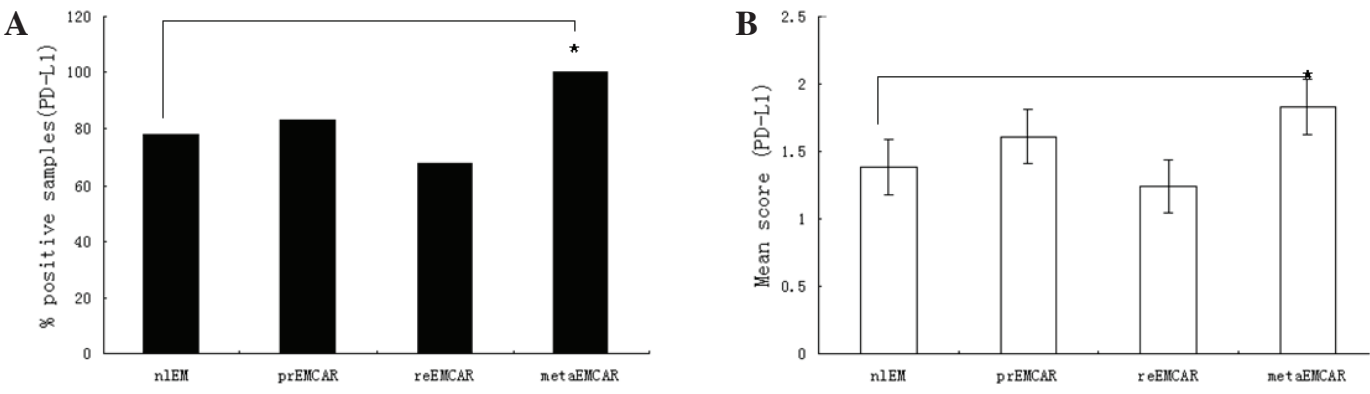

Figure 2. Expression of programmed death-ligand 1 (PD-L1) in endometrial carcinoma. (A) Positive rates and; (B) relative expression levels of PD-L1 in nlEM, normal endometrium; prEMCAR, primary endometrial carcinoma; recEMCAR, recurrent endometrial carcinoma; and metaEMCAR, metastatic endometrial carcinoma. ${ }^{*} \mathrm{P}<0.05$ vs. the control.
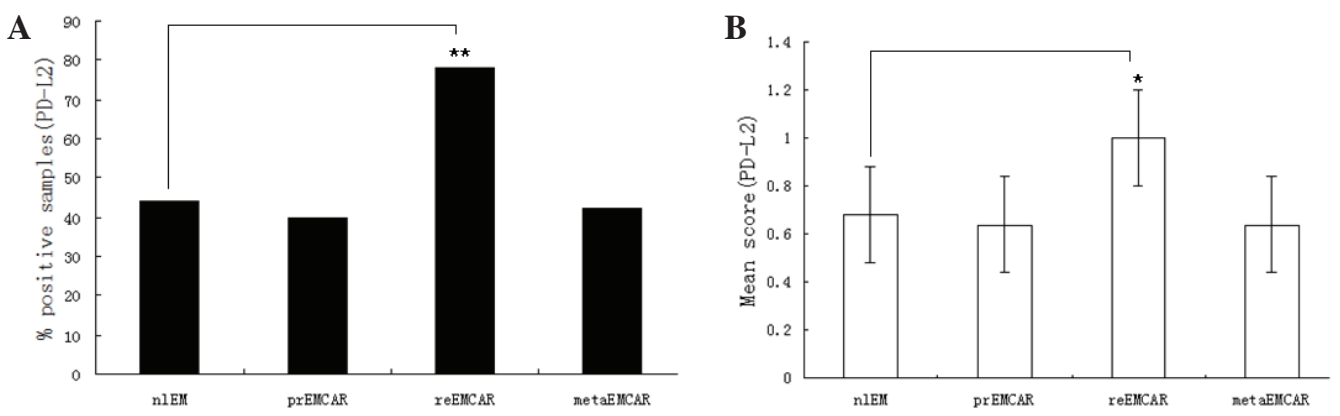

Figure 3. Expression of programmed death-ligand 2 (PD-L2) in endometrial carcinoma. (A) Positive rates and; (B) relative expression levels of PD-L2 in nlEM, normal endometrium; prEMCAR, primary endometrial carcinoma; recEMCAR, recurrent endometrial carcinoma; and metaEMCAR, metastatic endometrial carcinoma. ${ }^{*} \mathrm{P}<0.05$ and ${ }^{* * *} \mathrm{P}<0.01$ vs. the control.
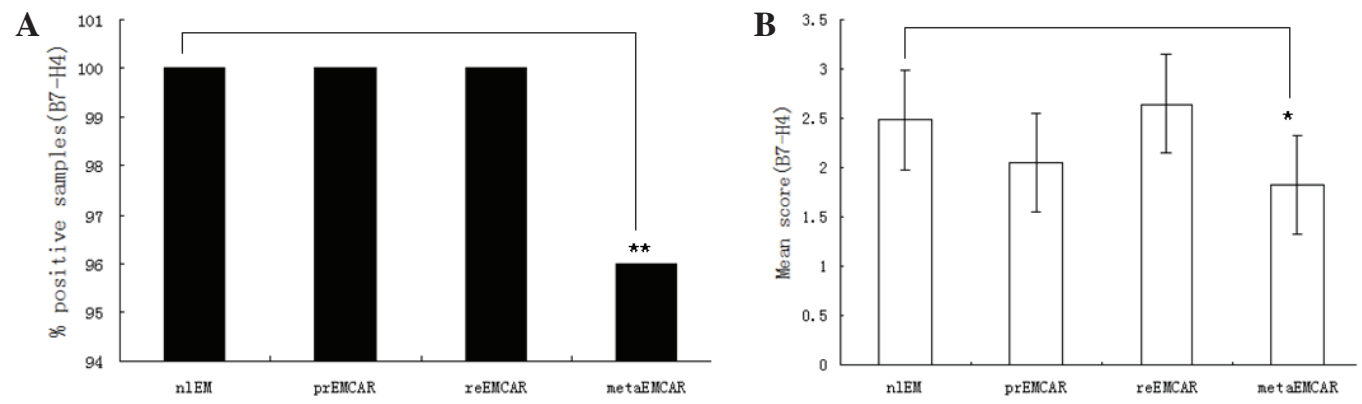

Figure 4. Expression of B7-H4 in endometrial carcinoma. (A) Positive rates and (B) relative expression levels of B7-H4 in nlEM, normal endometrium; prEMCAR, primary endometrial carcinoma; recEMCAR, recurrent endometrial carcinoma; and metaEMCAR, metastatic endometrial carcinoma. "P<0.05 and ${ }^{* *} \mathrm{P}<0.01$ vs. the control.

and PD-L2 with gynecological tumors. In the present study, there were no significant differences between the expression levels of PD-L1 and PD-L2 in normal tissues, as compared with tumor tissues. Contrary to expectations, the results of the 
present study suggested that high expression levels of PD-L1 are associated with an overall longer survival rate. However, this phenomenon was similarly identified in previous studies of melanoma, Merkel cellular tumor and mismatch repair-proliferated rectal cancer (16-18). Interferon- $\gamma$ and $\mathrm{CD}^{+} \mathrm{T}$ cells are able to promote the upregulation of PD-L1; therefore, the expression of PD-L1 may reflect an ongoing endogenous anti-tumor immune response, which may represent a negative feedback loop dependent on the invasion of the immune response (9).

Previous studies suggested that PD-L2 is exclusively expressed in DCs and MФ $(19,20)$; however, it has since been demonstrated that PD-L2 is expressed in somatic tissues and tumors, including tumor-associated fibroblasts (21), pharyngeal tissue (22) and hepatic cell carcinoma (23). The majority of biopsy specimens in the present study exhibited only low levels of PD-L2 expression, although there was a high rate of expression of PD-L2 in the recurrent endometrial carcinoma group. Previous studies have detected only low levels of PD-L2 in the majority of tumors $(4,24)$, which is concordant with the results of the present study. Furthermore, a previous study demonstrated that PD-L2 expression was negative in the majority of ovarian carcinomas (25). However, PD-L1 and PD-L2 have been detected in cervical cancer, although the results suggested that PD-L1 and PD-L2 were only expressed in a small number of tumors (26).

B7-H4 has been demonstrated to be expressed in various tumors, including breast and ovarian cancer (10); and previous research detected B7-H4 in ovarian tumor cells and tumor-associated MФ $(27,28)$. In the present study, the levels of B7-H4 were correlated with patient prognosis. Previous studies have detected B7-H4 expression in endometrial carcinoma $(29,30)$. Although these studies also detected B7-H4 expression in normal endometrium, B7-H4 staining demonstrated that its expression increased from normal to malignant endometrial carcinoma, which is inconsistent with the results of the present study, in which B7-H4 was found to be highly expressed in both benign and malignant tissue. This divergence in results may be due to the use of different antibodies, methods and scoring systems.

Galectin-1 and galectin-3 upregulation has previously been demonstrated in numerous tumors, including bladder cancer $(31,32)$, head and neck squamous cell carcinoma (33) and ovarian cancer $(34,35)$. Numerous studies have detected galectin-1 and galectin-3 expression in endometrial carcinoma, although the results have been conflicting $(4,36)$. van den Brûle et al (37) reported that the expression levels of galectin-1, but not galectin-3, were upregulated in endometrial carcinoma, as compared with normal endometrium. Similarly, galectin-1 expression levels have been demonstrated to be upregulated in undifferentiated cancer, as compared with differentiated cancer (38). Conversely, Brustmann et al (38) reported that the expression levels of galectin-3 were upregulated in endometrial carcinoma, as compared with normal endometrium, whereas Ege et al (39) reported that the expression levels of galectin-3 were decreased in endometrial carcinoma, as compared with normal endometrium. The results of the present study suggested that the expression levels of galectin-1 and galectin-3 were not significantly different between normal and tumor tissues.
The levels of galectin-1 in leiomyosarcoma tumor lysate have been shown to be significantly upregulated, as compared with that of the myometrium, tumor and other tissue types. Furthermore, the present study demonstrated galectin-3 expression in the membrane and cytoplasm of primary tumors, and the single cell suspension liquid of cervical cancer cell lines (26). The localization of galectin-3 has been reported to alter between various cancers (39). Brustmann et al (38) demonstrated increased expression of galectin-3 in the nucleus of endometrial carcinoma specimens, whereas Mylonas et al (40) reported galectin-3 expression in the cytoplasm and nucleus. However, the expression of galectin-3 in the cytoplasm has previously been associated with the invasion of the muscle layer near endometrial carcinoma (37), although this correlation was not demonstrated in the present study. Furthermore, the results of the present study did not demonstrate a difference in the expression levels of galectin-1 and galectin-3 between normal endometrium and endometrial carcinoma. This difference may be partially due to the use of divergent methods.

In conclusion, the present study reports the expression levels of immune checkpoint molecules PD-L1, PD-L2 and B7-H4, and the immunosuppressive molecules galectin-1 and galectin-3, in endometrial tumor specimens. The results of the present study, combined with positive reports from ongoing clinical trials $(4,41)$, suggest that inhibitors of these molecules may be considered as treatments for patients with endometrial tumors. Furthermore, the present study demonstrated that PD-L2 may be a useful target for immune suppression in a small number of patients with endometrial carcinoma, whereas both PD-L1 and B7-H4 were highly expressed in two of the analyzed tumor types, and therefore may be ideal candidate drug targets.

\section{References}

1. Nguyen TT, Hachisuga T, Urabe R, Kurita T, Kagami S, Kawagoe T, Shimajiri S and Nabeshima K: Significance of p53 expression in background endometrium in endometrial carcinoma. Virchows Arch 466: 695-702, 2015.

2. Murali R, Soslow RA and Weigelt B: Classification of endometrial carcinoma: More than two types. Lancet Oncol 15: e268-e278, 2014.

3. Sharp ZD and Bartke A: Evidence for down-regulation of phosphoinositide 3-kinase/Akt/mammalian target of rapamycin (PI3K/Akt/mTOR)-dependent translation regulatory signaling pathways in Ames dwarf mice. J Gerontol A Biol Sci Med Sci 60: 293-300, 2005

4. Vanderstraeten A, Luyten C, Verbist G, Tuyaerts S and Amant F: Mapping the immunosuppressive environment in uterine tumors: Implications for immunotherapy. Cancer Immunol Immunother 63: 545-557, 2014.

5. Kübler K, Ayub TH, Weber SK, Zivanovic O, Abramian A, Keyver-Paik MD, Mallmann MR, Kaiser C, Serçe NB, Kuhn W and Rudlowshi C: Prognostic significance of tumor-associated macrophages in endometrial adenocarcinoma. Gynecol Oncol 135: 176-183, 2014

6. Pardoll DM: The blockade of immune checkpoints in cancer immunotherapy. Nat Rev Cancer 12: 252-264, 2012.

7. Brahmer JR, Tykodi SS, Chow LQ, Hwu WJ, Topalian SL, Hwu P, Drake CG, Camacho LH, Kauh J, Odunsi K, et al: Safety and activity of anti-PD-L1 antibody in patients with advanced cancer. N Engl J Med 366: 2455-2465, 2012.

8. Topalian SL, Drake CG and Pardoll DM: Targeting the PD-1/B7-H1(PD-L1) pathway to activate anti-tumor immunity. Curr Opin Immunol 24: 207-212, 2012.

9. Topalian SL, Hodi FS, Brahmer JR, Gettinger SN, Smith DC, McDermott DF, Powderly JD, Carvajal RD, Sosman JA, Atkins MB, et al: Safety, activity, and immune correlates of anti-PD-1 antibody in cancer. N Engl J Med 366: 2443-2454, 2012. 
10. He C, Qiao H, Jiang $\mathrm{H}$ and Sun $\mathrm{X}$ : The inhibitory role of b7-h4 in antitumor immunity: Association with cancer progression and survival. Clin Dev Immunol 2011: 695834, 2011.

11. Yi KH and Chen L: Fine tuning the immune response through B7-H3 and B7-H4. Immunol Rev 229: 145-151, 2009.

12. Terness P, Kallikourdis M, Betz AG, Rabinovich GA, Saito S and Clark DA: Tolerance signaling molecules and pregnancy: IDO, galectins, and the renaissance of regulatory T cells. Am J Reprod Immunol 58: 238-254, 2007.

13. Ino K, Yoshida N, Kajiyama H, Shibata K, Yamamoto E, Kidokoro K, Takahashi N, Terauchi M, Nawa A, Nomura S, et al Indoleamine 2,3-dioxygenase is a novel prognostic indicator for endometrial cancer. Br J Cancer 95: 1555-1561, 2006.

14. de Jong RA, Kema IP, Boerma A, Boezen HM, van der Want JJ, Gooden MJ, Hollema H and Nijman HW: Prognostic role of indoleamine 2,3-dioxygenase in endometrial carcinoma. Gynecol Oncol 126: 474-480, 2012.

15. Kim JR, Moon YJ, Kwon KS, Bae JS, Wagle S, Kim KM, Park HS, Lee H, Moon WS, Chung MJ, et al: Tumor infiltrating PD1-positive lymphocytes and the expression of PD-L1 predict poor prognosis of soft tissue sarcomas. PLoS One 8: e82870, 2013.

16. Taube JM, Anders RA, Young GD, Xu H, Sharma R, McMiller TL, Chen S, Klein AP, Pardoll DM, Topalian SL and Chen L: Colocalization of inflammatory response with B7-h1 expression in human melanocytic lesions supports an adaptive resistance mechanism of immune escape. Sci Transl Med 4: 127ra37, 2012.

17. Droeser RA, Hirt C, Viehl CT, Frey DM, Nebiker C, Huber X, Zlobec I, Eppenberger-Castori S, Tzankov A, Rosso R, et al: Clinical impact of programmed cell death ligand 1 expression in colorectal cancer. Eur J Cancer 49: 2233-2242, 2013.

18. Lipson EJ, Vincent JG, Loyo M, Kagohara LT, Luber BS, Wang H, Xu H, Nayar SK, Wang TS, Sidransky D, et al: PD-L1 expression in the Merkel cell carcinoma microenvironment: Association with inflammation, Merkel cell polyomavirus and overall survival. Cancer Immunol Res 1: 54-63, 2013.

19. Huang Y, Zhao Y, Ran X and Wang C: Increased expression of herpesvirus entry mediator in 1,25-dihydroxyvitamin D3-treated mouse bone marrow-derived dendritic cells promotes the generation of $\mathrm{CD} 4^{+} \mathrm{CD} 25^{+} \mathrm{Foxp} 3^{+}$regulatory T cells. Mol Med Rep 9: 813-818, 2014.

20. Togno-Peirce C, Nava-Castro K, Terrazas LI and Morales-Montor J: Sex-associated expression of co-stimulatory molecules CD80, CD86, and accessory molecules, PDL-1, PDL-2 and MHC-II, in F480+ macrophages during murine cysticercosis. Biomed Res Int 2013: 570158, 2013.

21. Rozali EN, Hato SV, Robinson BW, Lake RA and Lesterhuis WJ: Programmed death ligand 2 in cancer-induced immune suppression. Clin Dev Immunol 2012: 656340, 2012.

22. Ohigashi Y, Sho M, Yamada Y, Tsurui Y, Hamada K, Ikeda N, Mizuno T, Yoriki R, Kashizuka H, Yane K, et al: Clinical significance of programmed death-1 ligand-1 and programmed death-1 ligand-2 expression in human esophageal cancer. Clin Cancer Res 11: 2947-2953, 2005.

23. Gao Q, Wang XY, Qiu SJ, Yamato I, Sho M, Nakajima Y, Zhou J, Li BZ, Shi YH, Xiao YS, et al: Overexpression of PD-L1 significantly associates with tumor aggressiveness and postoperative recurrence in human hepatocellular carcinoma. Clin Cancer Res 15: 971-979, 2009.

24. Kojima M, Murata S, Mekata E, Takebayashi K, Jaffee EM and Tani T: Fusion protein of mutant B7-DC and Fc enhances the antitumor immune effect of GM-CSF-secreting whole-cell vaccine. J Immunother 37: 147-154, 2014.

25. Hamanishi J, Mandai M, Iwasaki M, Okazaki T, Tanaka Y, Yamaguchi K, Higuchi T, Yagi H, Takakura K, Minato N, et al: Programmed cell death 1 ligand 1 and tumor-infiltrating CD8+ $\mathrm{T}$ lymphocytes are prognostic factors of human ovarian cancer. Proc Natl Acad Sci USA 104: 3360-3365, 2007.
26. Karim R, Jordanova ES, Piersma SJ, Kenter GG, Chen L, Boer JM, Melief CJ and van der Burg SH: Tumor-expressed B7-H1 and B7-DC in relation to PD-1+ T-cell infiltration and survival of patients with cervical carcinoma. Clin Cancer Res 15: 6341-6347, 2009.

27. Cheng L, Jiang J, Gao R, Wei S, Nan F, Li S and Kong B: B7-H4 expression promotes tumorigenesis in ovarian cancer. Int J Gynecol Cancer 19: 1481-1486, 2009.

28. Chen C, Qu QX, Shen Y, Mu CY, Zhu YB, Zhang XG and Huang JA: Induced expression of B7-H4 on the surface of lung cancer cell by the tumor-associated macrophages: A potential mechanism of immune escape. Cancer Lett 317: 99-105, 2012.

29. Miyatake T, Tringler B, Liu W, Liu SH, Papkoff J, Enomoto T, Torkko KC, Dehn DL, Swisher A and Shroyer KR: B7-H4 (DD-O110) is overexpressed in high risk uterine endometrioid adenocarcinomas and inversely correlated with tumor T-cell infiltration. Gynecol Oncol 106: 119-127, 2007.

30. Qian Y, Shen L, Cheng L, Wu Z and Yao H: B7-H4 expression in various tumors determined using a novel developed monoclonal antibody. Clin Exp Med 11: 163-170, 2011.

31. Cindolo L, Benvenuto G, Salvatore P, Pero R, Salvatore G, Mirone V, Prezioso D, Altieri V, Bruni CB and Chiariotti L: Galectin-1 and galectin-3 expression in human bladder transitional-cell carcinomas. Int J Cancer 84: 39-43, 1999.

32. Demydenko D and Berest I: Expression of galectin-1 in malignant tumors. Exp Oncol 31: 74-79, 2009.

33. Saussez S, Lorfevre F, Lequeux T, Laurent G, Chantrain G, Vertongen F, Toubeau G, Decaestecker C and Kiss R: The determination of the levels of circulating galectin-1 and -3 in HNSCC patients could be used to monitor tumor progression and/or responses to therapy. Oral Oncol 44: 86-93, 2008.

34. Kim MK, Sung CO, Do IG, Jeon HK, Song TJ, Park HS, Lee YY, Kim BG, Lee JW and Bae DS: Overexpression of Galectin-3 and its clinical significance in ovarian carcinoma. Int $\mathrm{J}$ Clin Oncol 16: 352-358, 2011.

35. van den Brûle F, Califice S, Garnier F, Fernandez PL, Berchuck A and Castronovo V: Galectin-1 accumulation in the ovary carcinoma peritumoral stroma is induced by ovary carcinoma cells and affects both cancer cell proliferation and adhesion to laminin-1 and fibronectin. Lab Invest 83: 377-386, 2003.

36. Stewart CJ and Crook ML: Galectin-3 expression in uterine endometrioid adenocarcinoma: comparison of staining in conventional tumor glands and in areas of MELF pattern myometrial invasion. Int J Gynecol Pathol 29: 555-561, 2010.

37. van den Brule FA, Buicu C, Berchuck A, Bast RC, Deprez M, Liu FT, Cooper DN, Pieters C, Sobel ME and Castronovo V: Expression of the 67-kD laminin receptor, galectin-1, and galectin-3 in advanced human uterine adenocarcinoma. Hum Pathol 27: 1185-1191, 1996.

38. Brustmann H, Riss D and Naudé S: Galectin-3 expression in normal, hyperplastic, and neoplastic endometrial tissues. Pathol Res Pract 199: 151-158, 2003

39. Ege CB, Akbulut M, Zekioğlu O and Ozdemir N: Investigation of galectin-3 and heparanase in endometrioid and serous carcinomas of the endometrium and correlation with known predictors of survival. Arch Gynecol Obstet 284: 1231-1239, 2011.

40. Mylonas I, Mayr D, Walzel H, Shabani N, Dian D, Kuhn C, Kunze S, Jeschke U and Friese K: Mucin 1, Thomsen-Friedenreich expression and galectin-1 binding in endometrioid adenocarcinoma: An immunohistochemical analysis. Anticancer Res 27: 1975-1980, 2007.

41. Howitt BE, Shukla SA, Sholl LM, Ritterhouse LL, Watkins JC, Rodig S, Stover E, Strickland KC, D'Andrea AD, Wu CJ, et al: Association of polymerase e-mutated and microsatellite-instable endometrial cancers with neoantigen load, number of tumor-Infiltrating lymphocytes, and expression of PD-1 and PD-L1. JAMA Oncol: Jul 9, 2015 (Epub ahead of print). 